Geometry $\mathcal{E}$ Topology

Volume 3 (1999) 1-20

Published: 15 March 1999

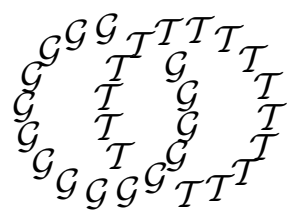

\title{
Contact Lie algebras of vector fields on the plane
}

\author{
Boris M DOUBrov \\ BORIS P KOMRAKOV \\ International Sophus Lie Centre \\ PO Box 70, 220123 Minsk, Belarus
}

Email: Doubrov@islc.minsk.by and Komrakov@islc.minsk.by

\begin{abstract}
The paper is devoted to the complete classification of all real Lie algebras of contact vector fields on the first jet space of one-dimensional submanifolds in the plane. This completes Sophus Lie's classification of all possible Lie algebras of contact symmetries for ordinary differential equations. As a main tool we use the abstract theory of filtered and graded Lie algebras. We also describe all differential and integral invariants of new Lie algebras found in the paper and discuss the infinite-dimensional case.
\end{abstract}

AMS Classification numbers Primary: 17B66, 53C30

Secondary: $34 \mathrm{~A} 26,58 \mathrm{~A} 20$

Keywords: Contact vector fields, filtered and graded Lie algebras, differential invariants

Proposed: Frances Kirwan

Seconded: Simon Donaldson, Robion Kirby
Received: 19 May 1998

Revised: 27 November 1998

Copyright Geometry and Topology 


\section{Introduction}

The problem of describing all finite-dimensional Lie algebras of vector fields is a starting point for the symmetry analysis of ordinary differential equations, because, having solved this problem, one finds all possible algebras of contact symmetries for ordinary differential equations.

Over the complex numbers this classification was done at the end of the last century by Sophus Lie [5]. He showed that, with three exceptions, all Lie algebras of contact vector fields, viewed up to equivalence, are lifts of Lie algebras of vector fields on the plane. The largest algebra of the three exceptions (so-called irreducible algebras of contact vector fields) is the algebra of contact symmetries of the equation $y^{\prime \prime \prime}=0$ and is isomorphic to $\mathfrak{s p}(4, \mathbb{C})$, while the other two are its subalgebras of dimension 6 and 7 .

In this paper we show that the problem of describing algebras of vector fields can be formulated in a natural way in terms of filtered and graded Lie algebras. This allows not only to give a new up-to-date proof of Sophus Lie's classification, which is as yet missing in the literature, but also to solve this problem over the field of real numbers. It turns out that in the real case there are 8 irreducible contact Lie algebras of vector fields on the plane, and one of them involves an arbitrary parameter.

Lie algebras of vector fields on the plane were also classified (both in real and complex case) by Sophus Lie [4], so that the description of irreducible Lie algebras of vector fields on the plane which is given in the present paper, basically concludes the description of all finite-dimensional contact Lie algebras of vector fields over the field of real numbers.

It should be noted that the problem of finding all irreducible contact Lie algebras over the real numbers was also considered by F. Engel in [1], which is mentioned in Sophus Lie's three-volume treatise [5] (volume 3, chapter 29, pages 760-761). P Olver, in his recently published book [7], cites this problem as unsolved.

\section{Jet space}

\subsection{Contact vector fields}

Let $M=J^{1}(\mathbb{R}, \mathbb{R})$ be the set of 1 -jets of mappings from $\mathbb{R}$ to $\mathbb{R}$, and let $\pi$ denote the natural projection $J^{1}(\mathbb{R}, \mathbb{R}) \rightarrow \mathbb{R}^{2}$. We fix a coordinate system 
$(x, y, z)$ on $M$ in which the 1 -jet of the mapping $f: \mathbb{R} \rightarrow \mathbb{R}$ at the point $x_{0}$ has the coordinates $\left(x_{0}, f\left(x_{0}\right), f^{\prime}\left(x_{0}\right)\right)$. The projection $\pi$ has in these coordinates the form $\pi:(x, y, z) \mapsto(x, y)$.

We can introduce a natural contact structure $M$. Indeed, there is a twodimensional distribution $C$ on $M$ which is not completely integrable and has the property that all its integral curves whose projection onto the plane is diffeomorphic, are precisely the curves of the form $\left(x, f(x), f^{\prime}(x)\right)$ with $f \in C^{\infty}(I)$, $I \subset \mathbb{R}$. In terms of coordinates, this distribution is given by the vector fields $\frac{\partial}{\partial z}$ and $\frac{\partial}{\partial x}+z \frac{\partial}{\partial y}$ or, alternatively, by the differential 1 -form $\omega=d y-z d x$. A (local) diffeomorphism $\phi$ of the manifold $M$ is said to be contact if $\phi$ preserves the contact distribution $C$, ie, if $d_{p} \phi\left(C_{p}\right)=C_{\phi(p)}$ for all $p \in M$. A vector field on $M$ is called contact if it generates a local one-parameter transformation group that consists solely of contact diffeomorphisms. It is easy to show that a vector field $X$ is contact if and only if $L_{X} \omega=\lambda \omega$ for some smooth function $\lambda$.

If $X$ is a contact vector field, then the function $f=\omega(X)$ is called the characteristic function of $X$. It completely determines the field $X$, which in this case is denoted by $X_{f}$ and has the form

$$
X_{f}=-\frac{\partial f}{\partial z} \frac{\partial}{\partial x}+\left(f-z \frac{\partial f}{\partial z}\right) \frac{\partial}{\partial y}+\left(\frac{\partial f}{\partial x}+z \frac{\partial f}{\partial y}\right) \frac{\partial}{\partial z} .
$$

The mapping $f \mapsto X_{f}$ establishes an isomorphism between the space of all smooth functions and that of contact vector fields on $M$. This allows to make the space $C^{\infty}(M)$ into a Lie algebra by letting $\{f, g\}=\omega\left(\left[X_{f}, X_{g}\right]\right)$.

\subsection{Prolongation operations}

If $\phi$ is a (local) diffeomorphism of the plane, then there exists a unique local contact transformation $\phi^{(1)}: J^{1}(\mathbb{R}, \mathbb{R}) \rightarrow J^{1}(\mathbb{R}, \mathbb{R})$ such that the following diagram is commutative:

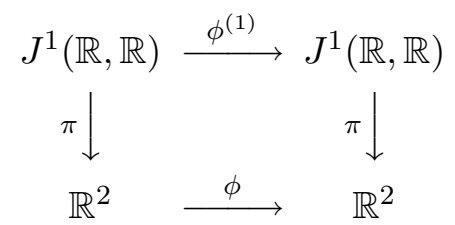

The transformation $\phi^{(1)}$ is then called the (first) prolongation of the diffeomorphism $\phi$ and, in terms of coordinates, has the from

$$
\phi^{(1)}:(x, y, z) \mapsto\left(A(x, y), B(x, y), \frac{B_{x}+B_{y} z}{A_{x}+A_{y} z}\right) .
$$


Similarly, for any vector field $X$ on the plane there exists a unique contact vector field $X^{(1)}$ on $J^{1}(\mathbb{R}, \mathbb{R})$ such that $\pi_{*}\left(X^{(1)}\right)=X$. This vector field $X^{(1)}$ is called the (first) prolongation of the vector field $X$ and has the form

$$
X^{(1)}=A(x, y) \frac{\partial}{\partial x}+B(x, y) \frac{\partial}{\partial y}+\left(B_{y} z^{2}+\left(B_{x}-A_{y}\right) z-A_{x}\right) \frac{\partial}{\partial z} .
$$

Its characteristic function is $B(x, y)-A(x, y) z$.

The mapping $X \mapsto X^{(1)}$ is an embedding of the Lie algebra of vector field on the plane into the Lie algebra of contact vector fields on $J^{1}(\mathbb{R}, \mathbb{R})$. The contact vector fields that lie in the image of this mapping are called point contact vector fields. Point vector fields $Y$ are characterized by the following two equivalent properties:

(1) any point vector field $Y$ is an infinitesimal symmetry of the vertical distribution $V$ on $J^{1}(\mathbb{R}, \mathbb{R})\left(V_{p}=\operatorname{ker} d_{p} \pi\right)$;

(2) the characteristic function of $Y$ is linear in $z$.

\subsection{Reducible Lie algebras of contact vector fields}

Definition A Lie algebra $\mathfrak{g}$ of contact vector fields is called reducible if there is a local contact diffeomorphism $\phi$ such that the Lie algebra $\phi_{*}(\mathfrak{g})$ consists only of point vector fields. Otherwise, $\mathfrak{g}$ is said to be irreducible.

Theorem 1 A Lie algebra $\mathfrak{g}$ of contact vector fields is irreducible if and only if it preserves no one-dimensional subdistribution of the contact distribution.

Proof Every Lie algebra that consists of point vector fields preserves the vertical distribution $V$, which is a one-dimensional subdistribution of the contact distribution $C$. Consequently, any reducible Lie algebra of contact vector fields also preserves a one-dimensional subdistribution of $C$.

Conversely, let $\mathfrak{g}$ be a Lie algebra of vector fields that preserves some onedimensional subdistribution $E$ of the contact distribution. If $A$ and $B$ are two functionally independent first integrals of $E$, then, as one can easily verify, the local diffeomorphism

$$
\phi:(x, y, z) \mapsto\left(A, B, \frac{B_{z}}{A_{z}}=\frac{B_{x}+z B_{y}}{A_{x}+z A_{y}}\right)
$$

is contact and transforms the vertical distribution $V$ to $E$. It follows that the Lie algebra $\phi_{*}^{-1}(\mathfrak{g})$ preserves the vertical distribution and hence consists of point vector fields. 
Corollary Any irreducible Lie algebra of contact vector fields is transitive at a point in general position.

Proof Let $\mathfrak{g}$ be an irreducible Lie algebra of contact vector fields. For an arbitrary point $p \in J^{1}(\mathbb{R}, \mathbb{R})$, we let $\mathfrak{g}(p)=\left\{X_{p} \mid X \in \mathfrak{g}\right\}$ and define $r=$ $\max _{p \in J^{1}(\mathbb{R}, \mathbb{R})} \operatorname{dim} \mathfrak{g}(p)$ and $U=\left\{p \in J^{1}(\mathbb{R}, \mathbb{R}) \mid \operatorname{dim} \mathfrak{g}(p)=r\right\}$. Then $U$ is obviously an open subset in $J^{1}(\mathbb{R}, \mathbb{R})$.

The Lie algebra $\mathfrak{g}$ is transitive at point in general position if and only if $r=3$. Assume the contrary. Then the subspaces $\mathfrak{g}(p)$ form a completely integrable distribution $E$ in $U$ which is invariant under $\mathfrak{g}$. Consider the following two possibilities:

$1^{\circ}: r=2$ Then the intersection $E_{p} \cap C_{p}$ is one-dimensional at the points in general position, and this family of subspaces forms a one-dimensional subdistribution of the contact distribution which is invariant under $\mathfrak{g}$.

$2^{\circ}: r<2$ In this case $E$ can be locally embedded into a two-dimensional completely integrable distribution which, as follows from its construction, is also invariant under $\mathfrak{g}$. Then, arguing as in the previous case, we conclude that the Lie algebra $\mathfrak{g}$ preserves a one-dimensional subdistribution of the contact distribution.

In this paper we restrict ourselves to a local description of finite-dimensional Lie algebras of contact vector fields at a point in general position. In particular, from now on we shall assume that all irreducible algebras of contact vector fields are transitive.

\section{An algebraic model of contact homogeneous space}

Let $\mathfrak{g}$ be a transitive Lie algebra of contact vector fields on $M=J^{1}(\mathbb{R}, \mathbb{R})$, let $o$ be an arbitrary point in $J^{1}(\mathbb{R}, \mathbb{R})$, and let $\mathfrak{g}_{0}=\mathfrak{g}_{o}$ be the subalgebra of $\mathfrak{g}$ that consists of all vector fields in $\mathfrak{g}$ vanishing at the point $o$. It is easy to show that the subalgebra $\mathfrak{g}_{0}$ is effective, ie, contains no nonzero ideals of $\mathfrak{g}$ (see, for example, [3, Theorem 10.1]).

We can identify $T_{o} M$ with $\mathfrak{g} / \mathfrak{g}_{0}$ in the obvious way. Then $C_{o}$ is identified with a certain submodule $W$ of the $\mathfrak{g}_{0}$-module $\mathfrak{g} / \mathfrak{g}_{0}$. Since the distribution $C$ is not completely integrable, the subspace $\left\{x \in \mathfrak{g} \mid x+\mathfrak{g}_{0} \in W\right\}$ will not be closed with respect to the multiplication in $\mathfrak{g}$. 
We define a decreasing chain of subspace in $\mathfrak{g}$ as follows: $\mathfrak{g}_{p}=\mathfrak{g}$ for all $p \leq-2$,

$$
\mathfrak{g}_{-1}=\left\{x \in \mathfrak{g} \mid x+\mathfrak{g}_{0} \in W\right\}, \quad \mathfrak{g}_{p+1}=\left\{x \in \mathfrak{g}_{p} \mid\left[x, \mathfrak{g}_{-1}\right] \subset \mathfrak{g}_{p}\right\} \text { for all } p \geq 0 .
$$

It is easily shown by induction that $\left[\mathfrak{g}_{p}, \mathfrak{g}_{q}\right] \subset \mathfrak{g}_{p+q}$ for all $p, q \in \mathbb{Z}$, so that the family of subspaces $\left\{\mathfrak{g}_{p}\right\}_{p \in \mathbb{Z}}$ defines a filtration of the Lie algebra $\mathfrak{g}$.

Definition A filtered Lie algebra $\mathfrak{g}$ is called a contact Lie algebra if

a) $\mathfrak{g}_{p}=\mathfrak{g}$ for all $p \leq-2$;

b) $\operatorname{codim}_{\mathfrak{g}} \mathfrak{g}_{-1}=1, \operatorname{codim}_{\mathfrak{g}} \mathfrak{g}_{0}=3$, and $\left[\mathfrak{g}_{-1}, \mathfrak{g}_{-1}\right]+\mathfrak{g}_{-1}=\mathfrak{g}$;

c) $\mathfrak{g}_{p+1}=\left\{x \in \mathfrak{g}_{p} \mid\left[x, \mathfrak{g}_{-1}\right] \subset \mathfrak{g}_{p}\right\}$ for all $p \geq 0$;

d) $\cap_{p \in \mathbb{Z}} \mathfrak{g}_{p}=\{0\}$.

Two contact Lie algebras are said to be isomorphic if they are isomorphic as filtered Lie algebras.

Show that any transitive Lie algebra $\mathfrak{g}$ of contact vector fields is a contact Lie algebra with respect to the above filtration. The properties a) and c) follow immediately from the way that the filtration in $\mathfrak{g}$ is introduced. Let us prove $b$ ). Since the contact distribution has codimension 1 , we get $\operatorname{codim}_{\mathfrak{g}} \mathfrak{g}_{-1}=1$. From transitivity of $\mathfrak{g}$ on $M$ we get $\operatorname{codim}_{\mathfrak{g}} \mathfrak{g}_{0}=3$. Next, since $C$ is not completely integrable, the subspace $\left[\mathfrak{g}_{-1}, \mathfrak{g}_{-1}\right]+\mathfrak{g}_{-1}$ is strictly greater than $\mathfrak{g}_{-1}$ and, hence, is equal to $\mathfrak{g}$.

Finally, Let $\mathfrak{a}=\cap_{p \in \mathbb{Z}} \mathfrak{g}_{p}$. Then, obviously, $\mathfrak{a}$ is an ideal in $\mathfrak{g}$ contained in $\mathfrak{g}_{0}$. Now since $\mathfrak{g}_{0}$ is an effective subalgebra, it follows that $\mathfrak{a}=\{0\}$. This proves $\mathrm{d}$ ).

Conversely, let $\mathfrak{g}$ be an arbitrary finite-dimensional contact Lie algebra. Then the pair $\left(\mathfrak{g}, \mathfrak{g}_{0}\right)$ determines a unique (up to local equivalence) realization of $\mathfrak{g}$ as a transitive Lie algebra of vector fields on $\mathbb{R}^{3}$. And the subspace $\mathfrak{g}_{-1}$ allows us to define a $\mathfrak{g}$-invariant two-dimensional distribution on $\mathbb{R}^{3}$ which is not completely integrable. Therefore, the Lie algebra $\mathfrak{g}$ admits a unique (up to local equivalence) realization as a transitive Lie algebra of contact vector fields.

Thus, the local classification of finite-dimensional transitive Lie algebras of contact vector fields on $J^{1}(\mathbb{R}, \mathbb{R})$ is equivalent to the classification (up to isomorphism) of the corresponding contact Lie algebras. Observe that the latter problem is algebraic and, as we shall see later, can be solved by purely algebraic means.

All $\mathfrak{g}$-invariant distributions on $J^{1}(\mathbb{R}, \mathbb{R})$ are in one-to-one correspondence with the submodules of the $\mathfrak{g}_{0}$-module $\mathfrak{g} / \mathfrak{g}_{0}$. In particular, the contact distribution corresponds to the submodule $\mathfrak{g}_{-1} / \mathfrak{g}_{0}$. From Theorem 1 now easily follows the next algebraic criterion for the irreducibility of $\mathfrak{g}$. 
Lemma 1 A transitive Lie algebra $\mathfrak{g}$ of contact vector fields is irreducible if and only if the $\mathfrak{g}_{0}$-module $\mathfrak{g}_{-1} / \mathfrak{g}_{0}$ is irreducible.

\section{Graded contact Lie algebras}

The major tool in the study of filtered Lie algebras is to consider graded Lie algebras associated with them. As we shall see later on, with a few exceptions, irreducible contact Lie algebras can be completely restored from their associated graded Lie algebras.

Definition A $\mathbb{Z}$-graded Lie algebra $\mathfrak{h}=\sum_{p \in \mathbb{Z}} \mathfrak{h}_{p}$ is called a graded contact Lie algebra if

a) $\mathfrak{h}_{p}=\{0\}$ for all $p<-2$;

b) $\operatorname{dim} \mathfrak{h}_{-1}=2, \operatorname{dim} \mathfrak{h}_{-2}=1$, and $\left[\mathfrak{h}_{-1}, \mathfrak{h}_{-1}\right]=\mathfrak{h}_{-2}$;

c) $\left\{x \in \mathfrak{h}_{p} \mid\left[x, \mathfrak{h}_{-1}\right]=0\right\}=\{0\}$ for all $p \geq 0$.

If $\mathfrak{g}$ is a contact Lie algebra, then it is clear that the associated graded Lie algebra $\mathfrak{h}=\sum_{p} \mathfrak{g}_{p} / \mathfrak{g}_{p+1}$ satisfies all three conditions in the above definition and is therefore a graded contact Lie algebra. Moreover, if $\mathfrak{g}$ is a filtered Lie algebra such that the associated graded Lie algebra $\mathfrak{h}$ is contact and $\cap_{p} \mathfrak{g}_{p}=\{0\}$, then it is easy to show that $\mathfrak{g}$ itself is a contact Lie algebra.

The concept of irreducibility for contact Lie algebras can be carried over to graded contact Lie algebras. From Lemma 1 it immediately follows that a contact Lie algebra $\mathfrak{g}$ is irreducible if and only if so is the $\mathfrak{h}_{0}$-module $\mathfrak{h}_{-1}$ in the corresponding graded contact Lie algebra $\mathfrak{h}$. The graded contact Lie algebras that satisfy this condition will be called irreducible.

The classification of all irreducible graded contact Lie algebras can be carried out using the methods developed in the works of Tanaka $[9,10]$. Slightly modifying the terminology of those papers (see also [2]), we introduce the concept of transitive graded Lie algebra, which generalizes the concept of graded contact Lie algebra.

Definition A graded Lie algebra $\mathfrak{g}=\oplus \mathfrak{g}_{p}$ is said to be transitive if it satisfies the following conditions:

(i) there exists a natural number $\mu \in \mathbb{N}$ such that $\mathfrak{g}_{-p}=\{0\}$ for all $p>\mu$;

(ii) $\left[\mathfrak{g}_{-1}, \mathfrak{g}_{-p}\right]=\mathfrak{g}_{-p-1}$ for all $p \geq 1$; 
(iii) if $x \in \mathfrak{g}_{p}$ for $p \geq 0$ and $\left[x, \mathfrak{g}_{-1}\right]=\{0\}$, then $x=0$.

It immediately follows from this definition that $\mathfrak{m}=\bigoplus_{p<0} \mathfrak{g}_{p}$ is the graded nilpotent Lie algebra generated by $\mathfrak{g}_{-1}$. Following Tanaka $[9,10]$, we shall call graded nilpotent Lie algebras of this kind fundamental. In particular, the fundamental nilpotent Lie algebra corresponding to a graded contact Lie algebra is none other than the three-dimensional Heisenberg algebra.

Let $\mathfrak{m}=\bigoplus_{p<0} \mathfrak{g}_{p}$ be an arbitrary fundamental graded Lie algebra. Then, as was shown by Tanaka [9], there exists a unique transitive graded Lie algebra $\mathfrak{g}(\mathfrak{m})=\bigoplus_{p \in \mathbb{Z}} \mathfrak{g}_{p}(\mathfrak{m})$ that satisfies the following conditions:

(1) $\mathfrak{g}_{p}(\mathfrak{m})=\mathfrak{g}_{p}$ for $p<0$;

(2) $\mathfrak{g}(\mathfrak{m})$ is the largest among all transitive graded Lie algebras satisfying condition (1).

This Lie algebra $\mathfrak{g}(\mathfrak{m})$ is called the (algebraic) extension of $\mathfrak{g}$. In particular, any transitive graded Lie algebra $\mathfrak{g}$ may be identified with a graded subalgebra of $\mathfrak{g}(\mathfrak{m})$, where $\mathfrak{m}=\bigoplus_{p<0} \mathfrak{g}_{p}$.

The Lie algebra $\mathfrak{g}(\mathfrak{m})$ has a clear geometrical meaning. Namely, let $M$ be a connected Lie group with Lie algebra $\mathfrak{m}$, and let $D$ be a left-invariant distribution on $M$ such that $D_{e}=\mathfrak{g}_{-1}$. Denote by $\mathcal{A}$ the Lie algebra of all germs of infinitesimal symmetries of $D$ at the identity element $e$ of $M$. Consider the following two subspaces in $\mathcal{A}$ :

$$
\begin{aligned}
\mathcal{A}_{0} & =\left\{\underline{X} \in \mathcal{A} \mid X_{e}=0\right\} \\
\mathcal{A}_{-1} & =\left\{\underline{X} \in \mathcal{A} \mid X_{e} \in D_{e}\right\}
\end{aligned}
$$

where $\underline{X}$ denotes the germ of the vector field $X$ at the point $e$. Now let

$$
\begin{aligned}
A_{-p-1} & =\left[\mathcal{A}_{-p}, \mathcal{A}_{-1}\right] \text { for all } p \geq 1 \\
A_{p} & =\left\{\xi \in \mathcal{A}_{p-1} \mid\left[\xi, \mathcal{A}_{-1}\right] \subset \mathcal{A}_{p-1}\right\} \text { for all } p \geq 1 .
\end{aligned}
$$

Then the family of subspaces $\left\{\mathcal{A}_{p}\right\}_{p \in \mathbb{Z}}$ forms a decreasing filtration of the Lie algebra $\mathcal{A}$, and $\mathfrak{g}(\mathfrak{m})$ can be identified with the associated graded algebra, ie, $\mathfrak{g}_{p}(\mathfrak{m}) \equiv \mathcal{A}_{p} / \mathcal{A}_{p+1}$ for all $p \in \mathbb{Z}$.

This geometrical interpretation allows to describe, without difficulty, the structure of $\mathfrak{g}(\mathfrak{m})$ in the case that we are interested in, namely in the case of graded contact Lie algebras. 
Let $\mathfrak{n}$ be the three-dimensional Heisenberg algebra and $\mathfrak{n}_{-2}=[\mathfrak{n}, \mathfrak{n}]$, while $\mathfrak{n}_{-1}$ is a two-dimensional subspace complementary to $[\mathfrak{n}, \mathfrak{n}]$. In this case we may assume without loss of generality that $D$ is precisely the contact distribution on $J^{1}(\mathbb{R}, \mathbb{R})$.

Using the description of all infinitesimal symmetries of the contact distribution, it is not hard to determine the structure of the Lie algebra $\mathfrak{g}(\mathfrak{n})$. It can be identified with the space of polynomials in $x, y, z$ with the bracket operation given by $X_{\{f, g\}}=\left[X_{f}, X_{g}\right]$. The space $\mathfrak{g}_{p}(\mathfrak{n})$ consists of all homogeneous polynomials of degree $p+2$, assuming that the variables $x, y, z$ are of degree 1,2 and 1 respectively. For example,

$$
\begin{aligned}
\mathfrak{g}_{-2}(\mathfrak{n}) & =\langle 1\rangle \\
\mathfrak{g}_{-1}(\mathfrak{n}) & =\langle x, z\rangle \\
\mathfrak{g}_{0}(\mathfrak{n}) & =\left\langle x^{2}, x z, z^{2}, y\right\rangle
\end{aligned}
$$

We shall now fix some fundamental graded Lie algebra $\mathfrak{m}$ and describe how one can classify all finite-dimensional graded subalgebras $\mathfrak{h}$ of the Lie algebra $\mathfrak{g}(\mathfrak{m})$ such that $\mathfrak{h}_{-p}=\mathfrak{m}_{-p}$ for all $p \geq 0$.

In what follows we shall always assume that $\mathfrak{h}_{-p}=\mathfrak{m}_{-p}=\mathfrak{g}_{-p}(\mathfrak{m})$ for all $p<0$. Suppose that for some $k \in \mathbb{N} \cup\{0\}$ we have a collection of subspaces $\mathfrak{h}_{i} \subset \mathfrak{g}_{i}(\mathfrak{m})$, $i=0, \ldots, k$, such that $\left[\mathfrak{h}_{p}, \mathfrak{h}_{q}\right] \subset \mathfrak{h}_{p+q} \forall p, q \leq k, p+q \leq k$. Using induction, we define a sequence of subspaces $\mathfrak{h}_{k+1}, \mathfrak{h}_{k+2}, \ldots$ as follows:

$$
\mathfrak{h}_{p+1}=\left\{x \in \mathfrak{g}_{p+1}(\mathfrak{m}) \mid\left[x, \mathfrak{h}_{-1}\right] \subset \mathfrak{h}_{p}\right\}
$$

for all $p \geq k$. It can be easily shown that

$$
\mathfrak{g}\left(\mathfrak{m}, \mathfrak{h}_{0}, \ldots, \mathfrak{h}_{k}\right)=\bigoplus_{p \in \mathbb{Z}} \mathfrak{h}_{p}
$$

is a graded subalgebra of $\mathfrak{g}(\mathfrak{m})$. This subalgebra is called the extension of the collection $\left(\mathfrak{h}_{0}, \ldots, \mathfrak{h}_{k}\right)$. Note that $\mathfrak{g}\left(\mathfrak{m}, \mathfrak{h}_{0}, \ldots, \mathfrak{h}_{k}\right)$ is the largest of all graded subalgebras whose $i$ th grading space coincides with $\mathfrak{h}_{i}$ for all $i \leq k$.

One the other hand, we can associate $\left(\mathfrak{h}_{0}, \ldots, \mathfrak{h}_{k}\right)$ with the graded subalgebra $\tilde{\mathfrak{g}}\left(\mathfrak{m}, \mathfrak{h}_{0}, \ldots, \mathfrak{h}_{k}\right)$ generated by these subspaces.

Now let $\mathfrak{h}$ be an arbitrary graded subalgebra of $\mathfrak{g}(\mathfrak{m})$ such that $\mathfrak{h}_{-p}=\mathfrak{g}_{-p}(\mathfrak{m})$ for all $p>0$. Then, obviously, for any $k \geq 0$ we have

$$
\tilde{\mathfrak{g}}\left(\mathfrak{m}, \mathfrak{h}_{0}, \ldots, \mathfrak{h}_{k}\right) \subset \mathfrak{h} \subset \mathfrak{g}\left(\mathfrak{m}, \mathfrak{h}_{0}, \ldots, \mathfrak{h}_{k}\right) .
$$

Finally, notice that $\mathfrak{g}_{0}(\mathfrak{m})$ is precisely the algebra of all derivations of $\mathfrak{m}$ that preserve the grading (see [9]), and all the subspaces $\mathfrak{h}_{p} \subset \mathfrak{g}_{p}(\mathfrak{m})$ are invariant 
under the natural action of $\mathfrak{h}_{0}$ on $\mathfrak{g}_{p}(\mathfrak{m})$. Based on these remarks, the following algorithm for the classification of the desired kind of subalgebras in $\mathfrak{g}(\mathfrak{m})$ suggests itself.

Step I Describe, up to conjugation, all subalgebras $\mathfrak{h}_{0} \subset \mathfrak{g}_{0}(\mathfrak{m})=\operatorname{Der}(\mathfrak{m})$. Go to Step III.

Step II Suppose that for some $k \in \mathbb{N} \cup\{0\}$, a collection of subspaces $\mathfrak{h}_{i} \subset$ $\mathfrak{g}_{i}(\mathfrak{m}), i=0, \ldots, k$, is already constructed such that

$$
\begin{aligned}
& \text { (i) }\left[\mathfrak{h}_{p}, \mathfrak{h}_{q}\right] \subset \mathfrak{h}_{p+q} \quad \forall p, q \leq k, p+q \leq k \\
& \text { (ii) } \operatorname{dim} \tilde{\mathfrak{g}}\left(\mathfrak{m}, \mathfrak{h}_{0}, \ldots, \mathfrak{h}_{k}\right)<\infty .
\end{aligned}
$$

Let

$$
\begin{aligned}
\tilde{\mathfrak{g}}_{k+1}\left(\mathfrak{m}, \mathfrak{h}_{0}, \ldots, \mathfrak{h}_{k}\right) & =\bigoplus_{\substack{i+j=k+1 \\
1 \leq i, j \leq k}}\left[\mathfrak{h}_{i}, \mathfrak{h}_{j}\right] \\
\mathfrak{g}_{k+1}\left(\mathfrak{m}, \mathfrak{h}_{0}, \ldots, \mathfrak{h}_{k}\right) & =\left\{x \in \mathfrak{g}_{k+1}(\mathfrak{m}) \mid\left[x, \mathfrak{h}_{-1}\right] \subset \mathfrak{h}_{k}\right\} .
\end{aligned}
$$

At this point we describe all $\mathfrak{h}_{0}$-invariant subspaces $\mathfrak{h}_{k+1}$ in $\mathfrak{g}_{k+1}\left(\mathfrak{m}, \mathfrak{h}_{0}, \ldots, \mathfrak{h}_{k}\right)$ such that

$$
\begin{aligned}
& \text { (i) } \tilde{\mathfrak{g}}_{k+1}\left(\mathfrak{m}, \mathfrak{h}_{0}, \ldots, \mathfrak{h}_{k}\right) \subset \mathfrak{h}_{k+1} \\
& \text { (ii) } \operatorname{dim} \tilde{\mathfrak{g}}\left(\mathfrak{m}, \mathfrak{h}_{0}, \ldots, \mathfrak{h}_{k+1}\right)<\infty .
\end{aligned}
$$

Step III Find the subalgebras $\tilde{\mathfrak{g}}\left(\mathfrak{m}, \mathfrak{h}_{0}, \ldots, \mathfrak{h}_{k+1}\right)$ and $\mathfrak{g}\left(\mathfrak{m}, \mathfrak{h}_{0}, \ldots, \mathfrak{h}_{k+1}\right)$. If these subalgebras are not the same, go to Step II. If, however, they coincide, then

$$
\mathfrak{h}=\tilde{\mathfrak{g}}\left(\mathfrak{m}, \mathfrak{h}_{0}, \ldots, \mathfrak{h}_{k+1}\right)=\mathfrak{g}\left(\mathfrak{m}, \mathfrak{h}_{0}, \ldots, \mathfrak{h}_{k+1}\right)
$$

is one of the desired subalgebras.

Now we shall use this algorithm to classify all irreducible graded contact Lie algebras over the field of real numbers.

Theorem 2 Let $\mathfrak{n}$ denote the three-dimensional real Heisenberg algebra, considered as a graded Lie algebra, and let $\mathfrak{g}(\mathfrak{n})$ be the universal extension of $\mathfrak{n}$. Then any finite-dimensional irreducible graded contact Lie algebra $\mathfrak{h}$ is isomorphic to one and only one of the following subalgebras of $\mathfrak{g}(\mathfrak{n})$ :

$$
\begin{array}{ll}
1^{\circ} & \left\langle 1, x, y, z, x^{2}, x z, z^{2}, x(2 y-x z), z(2 y-x z),(2 y-x z)^{2}\right\rangle \\
2^{\circ} & \left\langle 1, x, y, z, x^{2}, x z, z^{2}\right\rangle
\end{array}
$$




$$
\begin{aligned}
3^{\circ} & \left\langle 1, x, z, x^{2}, x z, z^{2}\right\rangle \\
4^{\circ} & \left\langle 1, x, z, x^{2}+z^{2}, 2 y-x z, x\left(x^{2}+z^{2}\right)-2 z(2 y-x z), z\left(x^{2}+z^{2}\right)+2 x(2 y-\right. \\
& \left.x z),\left(x^{2}+y^{2}\right)^{2}+4(2 y-x z)^{2}\right\rangle \\
5^{\circ} & \left\langle 1, x, z, x^{2}+z^{2}, 2 y-x z\right\rangle \\
6^{\circ} & \left\langle 1, x, z, x^{2}+z^{2}+\alpha(2 y-x z)\right\rangle, \alpha \geq 0
\end{aligned}
$$

Proof Fix a basis $\{x, z\}$ in the space $\mathfrak{g}_{-1}(\mathfrak{n})$. Then the action of the elements of $\mathfrak{g}_{0}(\mathfrak{n})$ on $\mathfrak{g}_{-1}(\mathfrak{n})$ is given by the following matrices:

$$
x^{2} \mapsto\left(\begin{array}{ll}
0 & 2 \\
0 & 0
\end{array}\right), \quad x z \mapsto\left(\begin{array}{cc}
-1 & 0 \\
0 & 1
\end{array}\right), \quad z^{2} \mapsto\left(\begin{array}{cc}
0 & 0 \\
-2 & 0
\end{array}\right), \quad y \mapsto\left(\begin{array}{rr}
-1 & 0 \\
0 & 0
\end{array}\right) .
$$

Therefore, the Lie algebra $\mathfrak{g}_{0}(\mathfrak{n})$ may be identified with $\mathfrak{g l}(2, \mathbb{R})$, and the $\mathfrak{g}_{0}(\mathfrak{n})-$ module $\mathfrak{g}_{-1}(\mathfrak{n})$ with the natural $\mathfrak{g l}(2, \mathbb{R})$-module.

Lemma 2 Any irreducible subalgebra of $\mathfrak{g l}(2, \mathbb{R})$ is conjugate to one and only one of the following subalgebras:
(i) $\left\{\left(\begin{array}{cc}\beta x & -x \\ x & \beta x\end{array}\right) \mid x \in \mathbb{R}\right\}, \beta \geq 0$
(ii) $\left\{\left(\begin{array}{cc}x & y \\ -y & x\end{array}\right) \mid x, y \in \mathbb{R}\right\}$
(iii) $\mathfrak{s l}(2, \mathbb{R})$
(iv) $\mathfrak{g l}(2, \mathbb{R})$

Proof If a subalgebra of $\mathfrak{g l}(2, \mathbb{R})$ is nonsolvable, then it is either three-dimensional and coincides with $\mathfrak{s l}(2, \mathbb{R})$, or four-dimensional and is equal to the whole of $\mathfrak{g l}(2, \mathbb{R})$. Any solvable irreducible subalgebra is commutative. If it is onedimensional, then, as follows from the classification of real Jordan normal forms of $2 \times 2$ matrices, it is conjugate to the subalgebra (i). If $\mathfrak{g}$ is two-dimensional, it coincides with the centralizer of one of the Jordan normal forms, which implies that it is conjugate to the subalgebra (ii).

If we identify $\mathfrak{g l}(2, \mathbb{R})$ and $\mathfrak{g}_{0}(\mathfrak{n})$, the subalgebras listed in Lemma 2 are identified with the following subspaces $\mathfrak{h}_{0} \subset \mathfrak{g}_{0}(\mathfrak{n})$ :
(i) $\left\langle x^{2}+z^{2}+\alpha(2 y-x z)\right\rangle, \alpha=2 \beta \geq 0$
(ii) $\left\langle x^{2}+z^{2}, 2 y-x z\right\rangle$
(iii) $\left\langle x^{2}, z^{2}, 2 y-x z\right\rangle$
(iv) $\left\langle x^{2}, x z, z^{2}, y\right\rangle$

Consider separately each one of these cases:

(i) It is easily verified that in this case we have $\mathfrak{g}_{1}\left(\mathfrak{n}, \mathfrak{h}_{0}\right)=\{0\}$. Therefore $\mathfrak{h}=\mathfrak{n} \oplus \mathfrak{h}_{0}$, and we arrive at the algebra which is listed in the theorem under the number $6^{\circ}$. 
(ii) Here we have

$$
\mathfrak{g}_{1}\left(\mathfrak{n}, \mathfrak{h}_{0}\right)=\left\langle x\left(x^{2}+z^{2}\right)-2 z(2 y-x z), z\left(x^{2}+z^{2}\right)+2 x(2 y-x z)\right\rangle,
$$

and the action of the subalgebra $\mathfrak{h}_{0}$ on this space is irreducible. Therefore, the space $\mathfrak{h}_{1} \subset \mathfrak{g}_{1}\left(\mathfrak{n}, \mathfrak{h}_{0}\right)$ is either zero or coincides with the whole of $\mathfrak{g}_{1}(\mathfrak{n})$. In the former case we immediately find that $\mathfrak{h}=\mathfrak{n} \oplus \mathfrak{h}_{0}$ (subalgebra $5^{\circ}$ ). In the second case the subalgebras $\mathfrak{g}\left(\mathfrak{n}, \mathfrak{h}_{0}, \mathfrak{h}_{1}\right)$ and $\tilde{\mathfrak{g}}\left(\mathfrak{n}, \mathfrak{h}_{0}, \mathfrak{h}_{1}\right)$ coincide and are equal to the subalgebra $4^{\circ}$ of the theorem.

(iii) Here $\mathfrak{g}_{1}\left(\mathfrak{n}, \mathfrak{h}_{0}\right)=\left\langle x^{3}, x^{2} z, x z^{2}, z^{3}\right\rangle$, and the $\mathfrak{h}_{0}$-module $\mathfrak{g}_{1}\left(\mathfrak{n}, \mathfrak{h}_{0}\right)$ is irreducible. Hence either we have $\mathfrak{h}_{1}=\{0\}$ and then $\mathfrak{h}=\mathfrak{n} \oplus \mathfrak{h}_{0}\left(\right.$ subalgebra $3^{\circ}$ ), or $\mathfrak{h}_{1}=\mathfrak{g}_{1}\left(\mathfrak{n}, \mathfrak{h}_{0}\right)$. In the latter case, however, the space $\mathfrak{h}_{1}$ generates a finitedimensional subalgebra.

(iv) Here $\mathfrak{g}_{1}\left(\mathfrak{n}, \mathfrak{h}_{0}\right)=\mathfrak{g}_{1}(\mathfrak{n})$, and the $\mathfrak{h}_{0}$-module $\mathfrak{g}_{1}(\mathfrak{n})$ is a sum of two irreducible submodules $W_{1}$ and $W_{2}$ of the form

$$
W_{1}=\left\langle x^{3}, x^{2} z, x z^{2}, z^{3}\right\rangle, \quad W_{2}=\langle x(2 y-x z), z(2 y-x z)\rangle .
$$

The submodule $W_{1}$ generates a finite-dimensional subalgebra, so that either $\mathfrak{h}_{1}=\{0\}$ or $\mathfrak{h}_{1}=W_{2}$. In the former case $\mathfrak{h}=\mathfrak{n} \oplus \mathfrak{h}_{0}$ (subalgebra $2^{\circ}$ ), while in the latter the subalgebras $\mathfrak{g}\left(\mathfrak{n}, \mathfrak{h}_{0}, \mathfrak{h}_{1}\right)$ and $\tilde{\mathfrak{g}}\left(\mathfrak{n}, \mathfrak{h}_{0}, \mathfrak{h}_{1}\right)$ coincide and are equal to the subalgebra $1^{\circ}$ of the theorem.

\section{Classification of contact Lie algebras}

In order to classify all finite-dimensional irreducible contact Lie algebras, it will suffice to describe all filtered Lie algebras whose associated graded Lie algebras are listed in Theorem 2. To solve this latter problem, we shall need the following result.

Lemma 3 Let $\mathfrak{g}$ be a finite-dimensional filtered Lie algebra, and $\mathfrak{h}$ the associated graded Lie algebra. If there is an element $e \in \mathfrak{h}_{0}$ such that

$$
\left[e, x_{p}\right]=p x_{p} \quad \forall x_{p} \in \mathfrak{h}_{p}
$$

then $\mathfrak{h}$, viewed as a filtered Lie algebra, is isomorphic to $\mathfrak{g}$.

Proof Suppose $e=\bar{e}+\mathfrak{g}_{1}$ for some $\bar{e} \in \mathfrak{g}_{0}$. For every $p \in \mathbb{Z}$, consider the subspace

$$
\mathfrak{g}^{p}(\bar{e})=\{x \in \mathfrak{g} \mid[\bar{e}, x]=p x\} .
$$


It is easy to show that, $\mathfrak{g}_{p}=\mathfrak{g}^{p}(\bar{e}) \oplus \mathfrak{g}_{p+1}$ for all $p \in \mathbb{Z}$. Thus, the subspace $\mathfrak{g}^{p}(\bar{e})$ may be identified with $\mathfrak{h}_{p}$, and since $\left[\mathfrak{g}^{i}(\bar{e}), \mathfrak{g}^{j}(\bar{e})\right] \subset \mathfrak{g}^{i+j}(\bar{e})$, this identification is in agreement with the structure of the Lie algebras $\mathfrak{g}$ and $\mathfrak{h}$. Hence, we have found an isomorphism of the Lie algebras $\mathfrak{g}$ and $\mathfrak{h}$ which is compatible with their filtrations.

For the graded Lie algebras listed in Theorem 2 under the numbers $1^{\circ}, 2^{\circ}$, $4^{\circ}, 5^{\circ}$, we can choose $e$ to be equal to $x z-2 y$, as this element is contained in all of these algebras. Then, in view of Lemma 3, the description of the corresponding filtered Lie algebras in these four cases is trivial. Consider the remaining two cases $3^{\circ}$ and $6^{\circ}$.

$3^{\circ}$ Let $\mathfrak{h}$ be the graded Lie algebra that appears under the number $3^{\circ}$ in Theorem 2 , and let $\mathfrak{g}$ be a contact Lie algebra whose associated graded Lie algebra is isomorphic to $\mathfrak{h}$. Since $\mathfrak{g}_{1}=\{0\}$, the subalgebra $\mathfrak{g}_{0}$ can be identified with the subalgebra $\mathfrak{h}_{0}$, which is isomorphic to $\mathfrak{s l}(2, \mathbb{R})$. Consider the $\mathfrak{g}_{0}$-module $\mathfrak{g}$. It is completely reducible, and its decomposition into a sum of irreducible submodules has the form: $\mathfrak{g}=V_{-2} \oplus V_{-1} \oplus \mathfrak{g}_{0}$, where the submodule $V_{-2}$ is one-dimensional and is a complement of $\mathfrak{g}_{-1}$, while the submodule $V_{-1}$ is two-dimensional and complements $\mathfrak{g}_{0}$ in $\mathfrak{g}_{-1}$. Therefore, the submodules $V_{-p}$, $p=1,2$ can be identified with the subspaces $\mathfrak{h}_{-p}$ of the graded Lie algebra $\mathfrak{h}$, which allows to identify $\mathfrak{g}$ and $\mathfrak{h}$ as vector spaces.

The structure of the Lie algebra $\mathfrak{g}$ is completely determined by the mappings $\alpha: V_{-2} \times V_{-1} \rightarrow \mathfrak{g}$ and $\beta: V_{-1} \wedge V_{-1} \rightarrow \mathfrak{g}$ defined as restrictions of the bracket operation in $\mathfrak{g}$ to the corresponding subspaces. The Jacobi identity shows that these mappings are both $\mathfrak{g}_{0}$-invariant. Since the $\mathfrak{g}_{0}$-module $V_{-1} \wedge V_{-1}$ is onedimensional and trivial, we have $\operatorname{im} \beta \subset V_{-2}$. Similarly, $\operatorname{im} \alpha \subset V_{-1}$. Now, computing the Jacobi identity for the basis vectors of $V_{-2}$ and $V_{-1}$, we find that the mapping $\alpha$ is zero. Thus, the identification of the spaces $\mathfrak{g}$ and $\mathfrak{h}$ is in agreement with the Lie algebra structures of these spaces, so that the Lie algebra $\mathfrak{g}$ is isomorphic to $\mathfrak{h}$, viewed as a filtered Lie algebra.

$6^{\circ}$ As in the above case, we can identify $\mathfrak{g}_{0}$ and $\mathfrak{h}_{0}$. Now, since the $\mathfrak{h}_{0}-$ modules $\mathfrak{h}_{0}$ and $\mathfrak{h}_{-1}$ are not isomorphic for any value of $\alpha$, we conclude that $\mathfrak{g}_{-1}$ contains a $\mathfrak{g}_{0}$-invariant subspace $V_{-1}$ which is a complement of $\mathfrak{g}_{0}$. Choose a basis $\{e\}$ for $\mathfrak{g}_{0}$ and a basis $\left\{u_{1}, u_{2}\right\}$ for $V_{-1}$ in such a way that

$$
\begin{aligned}
& {\left[e, u_{1}\right]=\alpha u_{1}-u_{2}} \\
& {\left[e, u_{2}\right]=u_{1}+\alpha u_{2} .}
\end{aligned}
$$

Geometry and Topology, Volume 3 (1999) 
Then the elements $e, u_{1}, u_{2}$, together with the element $u_{3}=\left[u_{1}, u_{2}\right]$, will, obviously, form a basis of $\mathfrak{g}$, and $\left[e, u_{3}\right]=2 \alpha u_{3}$. Furthermore, checking the Jacobi identity, we find that in case $\alpha \neq 0$ we have $\left[u_{1}, u_{2}\right]=\left[u_{1}, u_{3}\right]=0$, and the Lie algebra $\mathfrak{g}$ is isomorphic to $\mathfrak{h}$, viewed as a filtered Lie algebra. If $\alpha=0$, we have $\left[u_{1}, u_{2}\right]=\beta u_{3}$ and $\left[u_{1}, u_{3}\right]=-\beta u_{2}$ for some $\beta \in \mathbb{R}$. Note that the parameters $\beta$ and $x^{2} \beta$ with $x \in \mathbb{R}^{*}$ give here isomorphic Lie algebras, whatever the value of $x$ may be. Therefore, up to isomorphism of contact Lie algebras we may assume that $\beta=0, \pm 1$. If $\beta=0$, we find that $\mathfrak{g}$ is again isomorphic to $\mathfrak{h}$, viewed as a filtered Lie algebra. If $\beta=1$ or $\beta=-1$, the Lie algebra $\mathfrak{g}$ is isomorphic to $\mathfrak{g l}(2, \mathbb{R})$ or $\mathfrak{u}(2)$ respectively, while the subalgebras $\mathfrak{g}$ can be written, under this identification, in matrix form as follows:

$$
\left(\begin{array}{cc}
x & x \\
-x & x
\end{array}\right), \quad x \in \mathbb{R}
$$

Summing up what has been said, we obtain the following result:

Theorem 3 Any finite-dimensional irreducible contact Lie algebra is isomorphic to one and only one of the following:

I any of the graded contact Lie algebras listed in Theorem 2, if they are viewed as filtered Lie algebras;

II.1 $\mathfrak{g}=\mathfrak{g l}(2, \mathbb{R})$, where $\mathfrak{g}_{p}=\{0\}$ for $p \geq 1$,

$$
\mathfrak{g}_{0}=\left\{\left(\begin{array}{cc}
x & x \\
-x & x
\end{array}\right) \mid x \in \mathbb{R}\right\}, \quad \mathfrak{g}_{-1}=\left\{\left(\begin{array}{cc}
x+y & x+z \\
z-x & x-y
\end{array}\right) \mid x, y, z \in \mathbb{R}\right\} ;
$$

II.2 $\mathfrak{g}=\mathfrak{u}(2)$, where $\mathfrak{g}_{p}=\{0\}$ for $p \geq 1$,

$$
\mathfrak{g}_{0}=\left\{\left(\begin{array}{cc}
x & x \\
-x & x
\end{array}\right) \mid x \in \mathbb{R}\right\}, \quad \mathfrak{g}_{-1}=\left\{\left(\begin{array}{cc}
x+i y & x+i z \\
i z-x & x-i y
\end{array}\right) \mid x, y, z \in \mathbb{R}\right\} .
$$

From now on, to refer to irreducible contact algebras of type I, we shall employ the notation I. $n$, where $n$ is the number of the corresponding graded contact Lie algebra in Theorem 2.

\section{Applications}

\section{1}

Now we shall find explicit representations in contact vector fields for the Lie algebras of vector fields described above. Note that the mapping $f \mapsto X_{f}$ that 
maps an arbitrary function $f$ of the variables $x, y, z$ into the vector field whose characteristic function is $f$, defines an embedding of the Lie algebra $\mathfrak{g}(\mathfrak{n})$ into the algebra of all contact vector fields. In this way we immediately obtain the explicit representations in vector fields for those contact algebras $\mathfrak{g}$ which are isomorphic to their corresponding graded algebras.

Below we list three different representations of the space of characteristic functions for each of the contact algebras II.1 and II.2:

$$
\begin{array}{ll}
\text { II.1 } & \text { (a) }\left\langle(2 y-x z)^{2}+1, x-z(2 y-x z), z+x(2 y-x z), x^{2}+z^{2}\right\rangle \\
& \text { (b) }\left\langle x^{2}+z^{2}, 2 x(2 y-x z)+z\left(x^{2}+z^{2}+4\right), 2 z(2 y-x z)-x\left(x^{2}+z^{2}+\right.\right. \\
& \left.4), 16+4(2 y-x z)^{2}+\left(x^{2}+z^{2}\right)^{2}\right\rangle \\
& \text { (c) }\left\langle 1, z, \sqrt{1+z^{2}} \operatorname{sh} x, \sqrt{1+z^{2}} \operatorname{ch} x\right\rangle \\
\text { II.2 } & \text { (a) }\left\langle(2 y-x z)^{2}+1, x+z(2 y-x z), z-x(2 y-x z), x^{2}+z^{2}\right\rangle \\
& \text { (b) }\left\langle x^{2}+z^{2}, 2 x(2 y-x z)+z\left(x^{2}+z^{2}-4\right), 2 z(2 y-x z)-x\left(x^{2}+z^{2}-\right.\right. \\
& \left.4), 16+4(2 y-x z)^{2}+\left(x^{2}+z^{2}\right)^{2}\right\rangle \\
& \text { (c) }\left\langle 1, z, \sqrt{1-z^{2}} \sin x, \sqrt{1-z^{2}} \cos x\right\rangle
\end{array}
$$

In particular, from the representations (a) and (b) it follows that these two algebras of contact vector fields can both be embedded into the 10-dimensional algebra I.1 and into the 8-dimensional algebra I.4. The representations (c) are notable for the fact that the characteristic functions here are independent of $y$.

\section{2}

Consider the set of all contact vector fields of the form $X_{f}$, where the function $f$ has the form $f=a y+g(x, z)$ with $a \in \mathbb{R}$ and $g$ being an arbitrary function of $x, z$. It is easy to show that this condition is equivalent to the requirement that $X_{f}$ be an infinitesimal symmetry of the one-dimensional distribution $E$ generated by the vector field $\frac{\partial}{\partial y}$. Thus we see that this space of vector fields forms an infinite-dimensional subalgebra $\mathfrak{S}$ of the Lie algebra of all contact vector fields.

Consider the projection $\pi: \mathfrak{S} \rightarrow \mathcal{D}\left(\mathbb{R}^{2}\right)$ given by

$$
X_{f}=-g_{z} \frac{\partial}{\partial x}+\left(g-z g_{z}\right) \frac{\partial}{\partial y}+\left(g_{x}+a z\right) \frac{\partial}{\partial z} \mapsto-g_{z} \frac{\partial}{\partial x}+\left(g_{x}+a z\right) \frac{\partial}{\partial z} .
$$

It is easily verified that this mapping is a homomorphism of Lie algebras whose kernel is one-dimensional and is generated by $X_{1}$, while its image coincides with 
the set of all vector fields on the plane that preserve, up to a constant factor, the volume form $\omega=d x \wedge d z$ on the plane:

$$
\pi(\mathfrak{S})=\left\{X \in \mathcal{D}\left(\mathbb{R}^{2}\right) \mid L_{x}(\omega)=\lambda \omega, \lambda \in \mathbb{R}\right\} .
$$

Thus, with every Lie algebra of contact vector fields that preserves a onedimensional distribution complementary to the contact one, we can associate a Lie algebra of vector fields on the plane. Conversely, the inverse image of any subalgebra of the Lie algebra (1) of vector fields on the plane is some Lie algebra of contact vector fields in the jet space.

Note that all irreducible Lie algebras of contact vector fields, except I.1 and I.4, preserve a one-dimensional distribution complementary to the contact distribution, and hence can be embedded into $\mathfrak{S}$. The corresponding Lie algebras of vector fields on the plane are as follows:

I.2 the Lie algebra corresponding to the group of affine transformations of the plane;

I.3 the Lie algebra corresponding to the group of equi-affine transformations of the plane (ie, area-preserving affine transformations);

I.5 the Lie algebra corresponding to the group of similitude transformations;

I.6 $\left\langle\frac{\partial}{\partial x}, \frac{\partial}{\partial z},(\beta x-z) \frac{\partial}{\partial x}+(x+\beta z) \frac{\partial}{\partial z}\right\rangle, \beta=\alpha / 2$ (if $\alpha=0$, this Lie algebra corresponds to the group of Euclidean transformations);

II.1 the Lie algebra corresponding to the group of all transformations of the hyperbolic plane;

II.2 the Lie algebra corresponding to the group of rotations of the sphere.

\section{3}

The above correspondence allows to describe without any difficulty all differential and integral invariants for all Lie algebras $\mathfrak{g}$ of contact vector fields that satisfy the following conditions:

(a) $\mathfrak{g}$ preserves a one-dimensional distribution complementary to the contact one;

(b) $\mathfrak{g} \ni X_{1}=\frac{\partial}{\partial y}$.

Indeed, let $\left(x, y_{0}=y, y_{1}=z, y_{2}, \ldots, y_{n}\right)$ be the standard coordinate system in the space $J^{n}\left(\mathbb{R}^{2}\right)$ of $n$th jets of curves on the plane. (See [7] for definition of jet spaces and notions of differential and integral invariants.) Denote by $\mathfrak{g}^{(n)}$ the 
$n$th prolongation of the Lie algebra $\mathfrak{g}$. It then follows from the condition (b) that the differential and integral invariants of $\mathfrak{g}$ that have the order $n$ are independent of $y$ and may be considered on the manifold of the trajectories of the vector field $X_{1}^{(n)}$. These trajectories are given by the equations $y=$ const and can be parametrized by the coordinates $\left(x, y_{1}, \ldots, y_{n}\right)$. Furthermore, it turns out that if $n \geq 2$, the action of the algebra $\mathfrak{g}^{(n)}$ on that quotient manifold is equivalent to the action of the Lie algebra $\pi(\mathfrak{g})^{(n-1)}$ on the space of $(n-1)$ th jets, and the mapping $J^{n}\left(\mathbb{R}^{2}\right) \rightarrow J^{(n-1)}\left(\mathbb{R}^{2}\right)$ that establishes this equivalence has the form:

$$
\left(x, y_{1}, \ldots, y_{n}\right) \mapsto\left(x, y_{0}, \ldots, y_{n-1}\right) .
$$

Therefore, all differential and integral invariants of $\mathfrak{g}$ may be derived from the invariants of $\pi(\mathfrak{g})$ by substituting $y_{i+1}$ instead of $y_{i}$ for $i \geq 0$.

We remark that Sophus Lie [4] found all invariants for those Lie algebras of vector fields on the plane that correspond to the cases I.2 and I.3. The invariants of the 10-dimensional irreducible Lie algebra I.1 were computed in [7] over the complex numbers, and they remain unchanged on passing to the real case. Now we shall specify nontrivial integral and differential invariants of the least order for the rest of irreducible contact Lie algebras of vector fields; all other invariants can be derived from these by means of differentiation (see [7]).

\begin{tabular}{|c|c|c|}
\hline & Differential invariant & Integral invariant \\
\hline I.4 & $\frac{P}{Q^{8 / 3}}$ & $\frac{Q^{1 / 3} d x}{y_{2}^{2}+1}$ \\
I.5 & $\frac{\left(1+y_{2}^{2}\right) y_{4}-3 y_{2} y_{3}^{2}}{y_{2}^{2}}$ & $\frac{y_{3} d x}{1+y_{2}^{2}}$ \\
I.6 & $\frac{y_{3} e^{-\beta \operatorname{arctg} y_{2}}}{\left(1+y_{2}^{2}\right)^{3 / 2}}$ & $e^{\beta \operatorname{arctg} y_{2}}\left(1+y_{2}\right)^{1 / 2} d x$ \\
II.1 & $\frac{\sqrt{1+y_{1}^{2}}\left(\left(1+y_{1}^{2}\right) y_{3}-3 y_{1} y_{2}^{2}-y_{1}\left(1+y_{1}^{2}\right)^{2}\right)}{\left(\left(1+y_{1}^{2}\right)^{2}+y_{2}^{2}\right)^{3 / 2}}$ & $\left(\frac{\left(1+y_{1}^{2}\right)^{2}+y_{2}^{2}}{1+y_{1}^{2}}\right)^{1 / 2} d x$ \\
II.2 & $\frac{\sqrt{1-y_{1}^{2}}\left(\left(1-y_{1}^{2}\right) y_{3}+3 y_{1} y_{2}^{2}+y_{1}\left(1-y_{1}^{2}\right)^{2}\right)}{\left(\left(1-y_{1}^{2}\right)^{2}+y_{2}^{2}\right)^{3 / 2}}$ & $\left(\frac{\left(1-y_{1}^{2}\right)^{2}+y_{2}^{2}}{1-y_{1}^{2}}\right)^{1 / 2} d x$ \\
\hline
\end{tabular}

where

$$
\begin{aligned}
P=\left(y_{2}^{2}+1\right)^{2}\left(Q D^{2}(Q)-\frac{7}{6} D(Q)\right)+2\left(y_{2}^{2}+1\right) y_{2} y_{3} Q D(Q)- \\
-\left(9\left(y_{2}^{2}+1\right) y_{2} y_{4}-\frac{1}{2}\left(9 y_{2}^{2}-19\right) y_{3}^{2}\right) Q^{2} \\
Q=9\left(y_{2}^{2}+1\right)^{2} y_{5}-90\left(y_{2}^{2}+1\right) y_{2} y_{3} y_{4}+5\left(27 y_{2}^{2}-5\right) y_{3}^{3} \\
D=\frac{\partial}{\partial x}+y_{1} \frac{\partial}{\partial y}+\cdots+y_{7} \frac{\partial}{\partial y_{6}} \quad \text { (the operator of total differentiation). }
\end{aligned}
$$

For the algebras II.1 and II.2, we have chosen here their representations in contact vector fields that appear earlier under the letter (c). 
Notice that all contact Lie algebras listed in the table above are reducible over the field of complex numbers. Hence, for each of these algebras there exists a certain complex analytic contact transformation which takes it to one of the known canonical forms for contact Lie algebras over $\mathbb{C}$. Thus, the inverse thatsformation (prolonged as many times as needed) brings known invariants to the invariants of the initial Lie algebra.

For example, the contact transformation

$$
T:(x, y, z):(x, y, z) \mapsto\left(x+i z,-2 i y+1 / 2\left(x^{2}+2 i x z+z^{2}\right), x-i z\right)
$$

takes the contact Lie algebra I.5 to the algebra with the following space (over $\mathbb{C})$ of characteristic functions:

$$
\langle 1, x, y, z, x z\rangle \text {. }
$$

This contact Lie algebra is reducible and is the first prolongation of the following Lie algebra of vector fields on the plain:

$$
\left\langle\frac{\partial}{\partial x}, x \frac{\partial}{\partial x}, y \frac{\partial}{\partial y}, \frac{\partial}{\partial y}, x \frac{\partial}{\partial y}\right\rangle \text {. }
$$

The differential invariants of the least order for this Lie algebra were computed already (see, for example, [7]) and have the form:

$$
\begin{array}{cc}
\text { differential invariant: } & \frac{y_{2} y_{4}}{y_{3}^{2}} \\
\text { integral invariant: } & \frac{y_{3}}{y_{2}} d x .
\end{array}
$$

The third prolongation of the inverse transformation $T^{-1}$ takes these invariants to those given in the table above. In the similar way we can compute invariants for other contact Lie algebras of vector fields given in the table.

\section{4}

Consider the problem of classifying those infinite-dimensional subalgebras in the Lie algebra of contact vector fields that correspond to Lie pseudo-groups of contact transformations (ie, those that can be defined with the help of a finite number of differential equations; see [2]). As in the finite-dimensional case, all these algebras can be naturally divided into two classes: reducible ones, which are actually extensions of infinite-dimensional Lie algebras of vector fields on the plane, and irreducible ones. Over the field of complex numbers all irreducible infinite-dimensional Lie algebras of contact vector fields were described by Sophus Lie [6], who showed that, apart from the Lie algebra of all contact 
vector fields, there exist exactly two infinite-dimensional irreducible subalgebras, namely, the Lie algebra $\mathfrak{S}=\left\{X_{a y+g(x, z)}\right\}$, which we already mentioned earlier, and its commutant $[\mathfrak{S}, \mathfrak{S}]=\left\{X_{g(x, z)}\right\}$.

The methods for the description of contact Lie algebras that have been developed in this paper, can be easily generalized to the infinite-dimensional case. In particular, with these Lie algebras we can again associate graded contact Lie algebras that can be embedded into the universal extension $\mathfrak{g}(\mathfrak{n})$ of the three-dimensional Heisenberg algebra. Let $\mathfrak{h}$ be an infinite-dimensional graded subalgebra in $\mathfrak{g}(\mathfrak{n})$ such that $\mathfrak{h}_{p}=\mathfrak{g}_{p}(\mathfrak{n})$ for $p<0$ and such that the $\mathfrak{h}_{0}$-module $\mathfrak{h}_{-1}$ is irreducible. Then all possible types of subalgebras $\mathfrak{h}_{0}$ over the real numbers are listed in Lemma 2. As follows from the proof of Theorem 2, in the cases (i) and (ii) the Lie algebra $\mathfrak{h}$ is finite-dimensional. The consideration of the remaining cases (iii) and (iv) is the same over the complex and real numbers, and gives the infinite-dimensional Lie algebras of contact vector fields described above. Thus the classification of irreducible infinite-dimensional Lie algebras of contact vector fields remains unchanged on passing from the complex to the real case.

\section{References}

[1] F Engel, Kleinere Beiträge zur Gruppentheorie, Nr VII, Leipziger Ber. (1892) S. $292 \mathrm{ff}$

[2] V Guillemin, S Sternberg, An algebraic model of transitive differential geometry, Bull. Amer. Math. Soc. 70 (1964) 16-47

[3] R Hermann, Sophus Lie's 1880 transformation group paper, from: "Lie groups: history, frontiers and applications", Volume I, Math Sci Press (1975)

[4] S Lie, Klassifikation und Integration von gewöhnlichen Differentialgleichungen zwischen $x, y$, die eine Gruppe von Transformationen gestatten, I-VI, from: "Gesamelte Abhandlungen", Volume 5, Teubner, Leipzig (1924) 240-310, 362$427,432-448$

[5] S Lie, F Engel, Theorie der Transformationsgruppen, Teubner, Leipzig, Bd.1 (1889) Bd. 2, (1890) Bd. 3 (1893)

[6] S Lie, Über unendliche kontinuierliche Gruppen, from: "Gesamelte Abhandlungen", Volume 6, Teubner, Leipzig (1924)

[7] P J Olver, Equivalence, Invariants, and Symmetry, Cambridge University Press, Cambridge (1995)

[8] A Onishchik, Topology of Transitive Transformation Groups, Johann Ambrosius Barth Verlag GmbH, Leipzig (1994) 
[9] N Tanaka, On differential systems, graded Lie algebras and pseudo-groups, J. Math. Kyoto Univ. 10 (1970) 1-82

[10] N Tanaka, On the equivalence problems associated with simple graded Lie algebras, Hokkaido Math. J. 8 (1979) 23-84 